\title{
Nonlocality in kinetic roughening
}

\author{
Sutapa Mukherji* \\ Institut für Theoretische Physik, Universitat zu Köln, D-50937 Köln, Germany \\ Somendra M. Bhattacharjed \\ Institute of Physics, Bhubaneswar 751 005, India
}

(February 24, 2018)

\begin{abstract}
We propose a phenomenological equation to describe kinetic roughening of a growing surface in presence of long range interactions. The roughness of the evolving surface depends on the long range feature, and several distinct scenarios of phase transitions are possible. Experimental implications are discussed.
\end{abstract}

05.40.+j,05.70.Ln,64.60.Ht,68.35.Fx

\begin{abstract}
"Suppose that we take a bin and gently and uniformly pour in granular material. As the material in the bin builds up we can identify a surface and ask the question, 'What is the magnitude of the fluctuation in the height of surface (measured from the base of the bin)?' Also of interest is the length scale of the surface fluctuations and how they behave dynamically as more material is added." [1] And thus was born the Edwards-Wilkinson (EW) model for surface growth - a solvable linear model at the heart of our current understanding of numerous growth processes. A relevant nonlinear term, added to this by Kardar-Parisi-Zhang (KPZ) [2 《], brought to light the nuances of growth phenomena to the extent that the KPZ equation very soon became a paradigm, in particular for dynamic phase transitions. The applicability of the KPZ equation seems to encompass length scales from atomic level to macroscopic phenomena of every day life, but still a specter is haunting the field : why is the KPZ behavior not observed [3]?
\end{abstract}

Many of the experimental situations however involve complex processes which beg to go beyond the idealization as pouring of noninteracting particles. This is especially true if medium or fluctuation induced interactions interfere with the process as for example in the several recently studied systems involving proteins, colloids or latex particles [5], or in sedimentation. The major interaction one has to reckon with, as detailed numerical computations suggest [9.10], is the long ranged hydrodynamic interaction. Are such long range interactions relevant for the roughness of the surface? This question, the absence of a formalism to handle such interactions in the growth process, and the elusiveness of the KPZ behavior, led us to propose a simple phenomenological model by focusing on the long range nature of the extra force.

We like to develop a Langevin equation type description where long range aspects can be simulated by a force at each point of the growing surface exerted by the particles away from it - a hint to go beyond a strict local description. In the linear EW model, the growth is along the global normal to the surface without any overhang. The height $h(\mathbf{r}, t)$ at point $\mathbf{r}$ and time $t$ satisfies the diffusion equation with an additional noise term. If, instead of the global, the local normal is favored, the KPZ $(\nabla h)^{2}$ term is needed [2]. This nonlinear term describes the lateral growth at a point as can be seen from the height profile [3, 4 . We now extend this physical interpretation and take the gradient (or its magnitude) as a measure of the local density of deposited particles. The long range effect is now incorporated by coupling these gradients at two different points. Based on this intuitive picture, the equation we propose is the following:

$$
\begin{aligned}
& \frac{\partial h(\mathbf{r}, t)}{\partial t}=\kappa \nabla^{2} h(\mathbf{r}, t)+\eta(\mathbf{r}, t)+ \\
& \frac{1}{2} \int d \mathbf{r}^{\prime} \vartheta\left(\mathbf{r}^{\prime}\right) \nabla h\left(\mathbf{r}+\mathbf{r}^{\prime}, t\right) \cdot \nabla h\left(\mathbf{r}-\mathbf{r}^{\prime}, t\right),
\end{aligned}
$$

where $\kappa$ is the diffusion constant for the particles on the surface, and $\eta$ is a random space time dependent white noise of zero mean and $\left\langle\eta(\mathbf{r}, t) \eta\left(\mathbf{r}^{\prime}, t^{\prime}\right)\right\rangle=2 \Delta \delta\left(\mathbf{r}-\mathbf{r}^{\prime}\right) \delta(t-$ $\left.t^{\prime}\right)$. The kernel $\vartheta(r)$ is of long range and, in principle, connected to the underlying interactions [11]. So, we take $\vartheta(\mathbf{r})$ to have a short range part $\lambda_{0} \delta(\mathbf{r})$ and a long range part $\sim r^{\rho-d}$, or more precisely, in Fourier space, $\vartheta(\mathbf{k})=\lambda_{0}+\lambda_{\rho} k^{-\rho}$. Eq. 11 then smoothly [12] goes over to the KPZ equation for $\lambda_{\rho}=0$. We show that this leading term introduced is sufficient to yield a new fixed point with continuously varying exponents, and different phase transitions not found in the KPZ problem. The connection with experiments is discussed near the end of this paper.

A central quantity of interest in growth problems is the scaling behavior of fluctuation of the height, \langle|$h(\mathbf{r}, t)-$ $\left.\left.h(\mathbf{0}, 0)\right|^{2}\right\rangle$ which on a large length and time scale has a scaling form $|\mathbf{r}|^{2 \chi} \mathcal{F}\left(|t| /|\mathbf{r}|^{z}\right)$. Here $\chi$ is the roughness exponent of the growing surface and $z$ is the dynamic exponent. These two exponents define the universality classes of roughening.

At $d=1$, for the local growth (i.e. KPZ) equation a disorder dominated rough phase is found for all $\lambda_{0}$ by several exact treatments [13,14 providing $\chi=1 / 2$ and 
$z=3 / 2$. The nonlinearity is marginally relevant at $d=2$ and for $d>2$ there is a phase transition from a strong disorder dominated phase $(\chi+z=2$ for all $d)$ to a weak coupling phase where nonlinearity is irrelevant, i.e. a flat phase with $z=2$. The perturbation theory is inadequate for the strong coupling phase at $d \geq 2$ due to the lack of a perturbative fixed point [15]. Numerical simulations [16] predict $z \approx 1.6$ at $d=2$. The phase transition is, however, under control, with $z=2 \forall d>2$ [17, 18], with a rather complicated critical behavior. [19,20].

A simple scaling analysis indicates that both $\lambda_{\rho}$ and $\lambda_{0}$ are relevant for $d<2$ at the Gaussian fixed point (EW) where one expects, $\chi=(2-d) / 2$ and $z=2$. This follows from the scale invariance of Eq.(11) under the transformation $r \rightarrow b r, t \rightarrow b^{z} t, h \rightarrow b^{\chi} h$, whence $\kappa \rightarrow b^{z-2} \kappa$, $\Delta \rightarrow b^{z-d-2 \chi} \Delta, \lambda_{0} \rightarrow b^{\chi+z-2} \lambda_{0}$ and $\lambda_{\rho} \rightarrow b^{z+\chi+\rho-2} \lambda_{\rho}$. Also for any nonzero $\lambda_{\rho}$ with $\rho>0$ the local KPZ theory $\left(\lambda_{\rho}=0\right.$, and $\left.\chi+z=2\right)$ is "unstable" under renormalization and a non-KPZ behavior is expected. For $2<d<2+2 \rho$ only $\lambda_{\rho}$ is relevant at the EW fixed point. In the following we adopt a dynamic renormalization group (RG )procedure. Our results show a new stable fixed point at $d=1$, for any $\rho>0$. Another interesting consequence of this nonlocality is the possibility of a stable fixed point at $d=2$ for a certain range of $\rho$. The marginal relevance of nonlinearity in the original KPZ theory is destroyed.

The renormalization procedure is most succinctly described through the Fourier modes momentum $\mathbf{q}$ and frequency $\omega$ in terms of which Eq. (11) becomes

$$
\begin{aligned}
& h(\mathbf{q}, \omega)=G_{0}(\mathbf{q}, \omega)\left[\eta(\mathbf{q}, \omega)-(1 / 2)(2 \pi)^{-d-1} \times\right. \\
& \left.\int d \mathbf{q}^{\prime} d \omega^{\prime} \vartheta\left(2 \mathbf{q}^{\prime}\right) \mathbf{q}_{+} \cdot \mathbf{q}_{-} h\left(\mathbf{q}_{+}, \omega_{+}\right) h\left(\mathbf{q}_{-}, \omega_{-}\right)\right],
\end{aligned}
$$

where, symbolically, $X_{ \pm}=X / 2 \pm X^{\prime}$ with $X=\mathbf{q}$ or $\omega$. Here $G_{0}(\mathbf{q}, \omega)=1 /\left(\kappa q^{2}-i \omega\right)$ represents the bare propagator or the Green function for the diffusion equation. We follow the usual iterative perturbation scheme where $h$ in the RHS of Eq. (2) is replaced by Eq. (2) itself upto $O\left(\vartheta^{2}\right)$. A convenient diagrammatic representation can be set up from this scheme and the renormalization of the various parameters can be obtained from appropriate vertex functions. We skip the details as they are very similar to Ref. [21]. In the subsequent renormalization procedure, we integrate out small length scale fluctuations over a momentum shell $\Lambda e^{-l} \leq q^{\prime} \leq \Lambda$ to obtain the effective parameters for a similar equation but with a smaller cutoff $\Lambda e^{-l}$, where $\Lambda$ (set to 1 ) is related to the microscopic cutoff. A subsequent rescaling then restores the cut off to $\Lambda$.

The effective propagator $G(\mathbf{q}, \omega) \equiv h(\mathbf{q}, \omega) / \eta(\mathbf{q}, \omega)$, gives the renormalization of tension $\kappa$. The effective noise, obtained from $\left\langle h^{*}(\mathbf{q}, \omega) h(\mathbf{q}, \omega)\right\rangle=$ $2 \tilde{\Delta} G(\mathbf{q}, \omega) G(-\mathbf{q},-\omega)$, gives the renormalization of the disorder. Next we look for the terms contributing to the effective nonlinearity. Note that the RG transformation, being analytic in nature, cannot generate a singular term to renormalize $\lambda_{\rho}$ for $\rho>-2$. In fact there is no renormalization of $\lambda_{0}$ either. A contribution to $\lambda_{0}$ could come from terms of $O\left(\Delta \vartheta^{3}\right)$, and a straightforward calculation [21] shows that such terms do cancel each other [22].

Following the above procedure we arrive at the flow equations for $\kappa$ and $\Delta$ as

$$
\begin{aligned}
& \frac{d \kappa}{d l}=\kappa\left[z-\left(2+\frac{\Delta K_{d}}{\kappa^{3}} \vartheta(2) \vartheta(1) \frac{(d-2)+3 f(1)}{4 d}\right)\right] \\
& \frac{d \Delta}{d l}=(z-d-2 \chi) \Delta+\frac{\Delta^{2} K_{d}}{4 \kappa^{3}} \vartheta(2)^{2},
\end{aligned}
$$

where $f(a)=\partial \ln \vartheta(k) /\left.\partial \ln k\right|_{k=a}$, the (effective) exponent of $\vartheta(k)$ and $K_{d}=S_{d} /(2 \pi)^{d}, S_{d}$ being the surface area of a $d$-dimensional unit sphere. The flow equations for $\lambda_{0}$ and $\lambda_{\rho}$, having contribution only from the rescaling, are $d \lambda_{x} / d l=(\chi+z-2+x) \lambda_{x}, \quad(x=0$ or $\rho)$. The two parameters $\chi$ and $z$ are chosen to keep $\kappa$ and one of $\lambda_{x}$ invariant.

In terms of $U_{x}^{2}=\Delta \lambda_{x}^{2} K_{d} / \kappa^{3}$, and $R=U_{0} / U_{\rho}$, with the choice $\chi+z=2$ (or $2-\rho$ ) and $z$ equal to the expression inside the big round bracket of Eq. 月, the flow equations can be combined into two as

$$
\frac{d U_{0}}{d l}=\frac{(2-d)}{2} U_{0}+\frac{2 d-3}{4 d} U_{0}^{3}+\frac{U_{0} U_{\rho}}{8 d}\left[c_{0} U_{0}+c_{1} U_{\rho}\right],
$$

and $d R / d l=-\rho R$, where $c_{0}=(5 d-6)\left(1+2^{-\rho}\right)-2 d-9 \rho$, and $c_{1}=\left[\left(3+2^{-\rho}\right) d-6-9 \rho\right] 2^{-\rho}$. The equation for $R$ rules out the existence of any off axis fixed point in the $U_{0}$ and $U_{\rho}$ parameter space ( except for $\rho=0$, when there is a trivial marginal fixed line.)

There are only two sets of axial fixed points, $\mathrm{SR} \equiv$ $\left\{U_{0}^{*^{2}}=2 d(d-2) /(2 d-3), U_{\rho}^{*^{2}}=0\right\}$, with $\chi+z=2$, and $\mathrm{LR} \equiv\left\{U_{0}^{*^{2}}=0, U_{\rho}^{*^{2}}=4 d(d-2-2 \rho) / c_{1}\right\}$ with $\chi+z=2-\rho$. The first set (SR), with $\lambda_{\rho}=0$, corresponds to the known KPZ fixed point, whose properties have already been mentioned. However we see a relevant perturbation $U_{\rho}$ which grows at this fixed point. The stable fixed point for $d<2+2 \rho$, with $\rho>0$ is LR, except for the region bounded by $d=(9 \rho+6) /\left(2^{-\rho}+3\right)$ and $d=2+2 \rho$. This excluded region is, like the KPZ case, an artifact of one loop renormalization 15]. At the new fixed point LR

$$
z=2+\Phi, \text { and } \chi=-\rho-\Phi,
$$

where $\left.\Phi=(d-2-2 \rho)(d-2-3 \rho) /\left[d\left(2^{-\rho}+3\right)-6-9 \rho\right)\right]$ This fixed point admits $z<1$ (not unexpected for long range cases) but by virtue of the relation $\chi+z=2-\rho$, $\chi$ need not be greater than 1 , a requirement for ignoring higher order terms in Eq. 1. At $d=2$, the marginal relevance of $U_{0}$ of the KPZ theory is lost and there is a stable fixed point (LR) for $\rho>0.194$. 


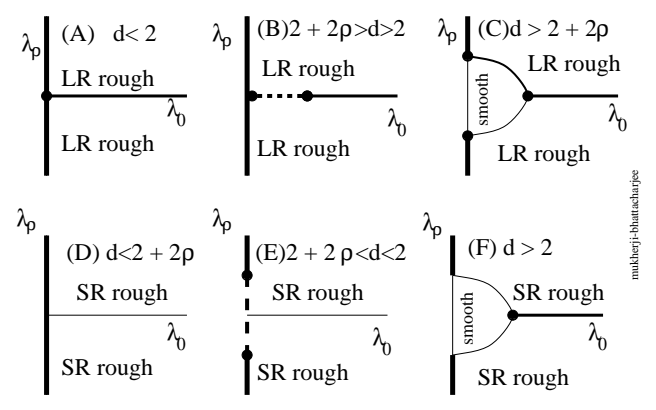

FIG. 1. $\lambda_{\rho}$ vs $\lambda_{0}$ phase diagram. (A)-(C) correspond to $\rho>0$, while (D)-(F) to $\rho<0$. Thick line along the y-axis represents the LR phase, while the medium thick line along the $\mathrm{x}$-axis SR phase. In (D) and (E) the phase is SR (KPZ) type for all $\lambda_{\rho} \neq 0$. The dashed line in (B) and (E) represents a smooth phase, which extends over a region in $(\mathrm{C})$ and $(\mathrm{F})$.

To discuss the surface morphology and the phase transitions [23], we consider different values of $d$ and $\rho>-2$. See fig. 1. Also note that the invariance of Eq. 1 under $h \rightarrow-h$ and $\lambda \rightarrow-\lambda$, is respected by the fixed point equations. Since the nonlinear term is like a force, the change in sign of $\lambda$ corresponds to a "push"- "pull" change or a growing to a receding surface case. We therefore consider both positive and negative values of $\lambda_{\rho}$, and without any loss in generality, take $\lambda_{0} \geq 0$.

Case I: $d<\min (2,2+2 \rho)$ : For $\rho>0$, the stable fixed point, if exists, is LR with the dynamic exponent given by Eq. 6. Even if it does not exist in this one loop approach, still, from the flow, the phase is the "strong" disorder type. We call this an LR phase to distinguish it from the SR or KPZ phase. It is possible to have a transition between two identical LR rough phases (pushpull). The critical behavior is EW type if there is strictly no short range nonlinearity else it is KPZ (SR) type. See Fig 1A. In contrast, for $\rho<0$ (Fig 1D), the LR is irrelevant and the surface behavior is always SR (KPZ) type except for $\lambda_{0}=0$, when it is an LR phase. There is no phase transition for $\lambda_{0} \neq 0$.

Case II: $\min (2,2+2 \rho)<d<\max (2,2+2 \rho)$ : The phases are LR or SR depending on the sign of $\rho$ (Fig $1 \mathrm{~B}, 1 \mathrm{E})$. For $\rho>0$, the critical behavior depends on the strength of the SR nonlinearity, $\lambda_{0}$. For small $\lambda_{0}<\lambda_{0 c}$, the critical surface is a smooth one while for $\lambda_{0}>\lambda_{0 c}$ it is KPZ. There is no transition if $\rho<0$ and $\lambda_{0} \neq 0$. However, for $\lambda_{0}=0$, there is a LR rough to smooth transition for $\rho<0$. See Fig. 1E.

Case III: $d>\max (2,2+2 \rho)$ : For $\rho>0$, the LR fixedpoint is unstable. A small nonlinearity dies down yielding a smooth surface while large nonlinearity will produce an LR rough phase. Unfortunately the absence of a fixed point forbids any prediction of the behavior of the LR phase. The unstable LR fixed-point controls the transition between the rough and the smooth surface with a dynamic exponent $z_{c}=2+c \rho \epsilon+O\left(\epsilon^{2}\right)$ where $\epsilon=d-2-2 \rho$ and $c=-2^{\rho} /\left[(2+2 \rho)\left(1+32^{\rho}\right)-32^{\rho}(2+3 \rho)\right]$. This is in striking contrast with the believed to be exact result of $z_{c}=2$ for the KPZ case. The phase diagram is shown in Fig $1 \mathrm{C}$. For $\rho<0$, there is a phase transition between a SR rough and a smooth phase only if $\lambda_{0}$ is less than a critical value as shown in Fig. 1F.

Experiments on colloids [5] have yielded a value of $\chi=.71$ which is also the value obtained from paper burning experiments [24]. These are taken as the exponent for a driven surface (line in the $d=1$ example). For the colloid problem, hydrodynamic interaction (HI) is important while in the paper burning experiment, it is possible to have a long range interaction through the microstructure of the paper. With this $\chi \mathrm{Eq}$. 6 at the LR fixed point in $d=1$ gives $\rho=-.12$. At this point, it is difficult to conclude if this is the transient exponent seen, eventually going over to the KPZ value on large length and time scales (Fig. 1D), or it is a true $\lambda_{0}=0$ case. Other cases where HI is known to play a role, namely the deposition of latex particles or proteins, the experiments have not been done for roughness of the growing surface. We believe such experiments will shed new light on growth phenomena.

For the KPZ problem, it is known that anisotropy of the substrate can lead to an overall irrelevance of the nonlinearity in two dimensions [25]. To see if anisotropy can have a major effect in the long range case, we now consider a variation of the problem where the long range interaction has different amplitudes in different directions. Restricting ourselves to $d=2$, we take

$$
\begin{gathered}
\frac{\partial h(\mathbf{r}, t)}{\partial t}=\kappa_{\|} \partial_{\|}^{2} h(\mathbf{r}, t)+\kappa_{\perp} \partial_{\perp}^{2} h(\mathbf{r}, t)+\eta(\mathbf{r}, t)+ \\
\sum_{\Psi=\|, \perp} \int d \mathbf{r}^{\prime} \frac{1}{2} \vartheta_{\Psi}\left(\mathbf{r}^{\prime}\right) \partial_{\Psi} h\left(\mathbf{r}+\mathbf{r}^{\prime}, t\right) \partial_{\Psi} h\left(\mathbf{r}-\mathbf{r}^{\prime}, t\right) .
\end{gathered}
$$

as the anisotropic version of Eq. 11. In the isotropic case, $r_{\lambda} \equiv \vartheta_{\|}(\mathbf{r}) / \vartheta_{\perp}(\mathbf{r})=1$ and $r_{\kappa} \equiv \kappa_{\|} / \kappa_{\perp}=1$ reproduce eqn 1. For simplicity let us concentrate only on a case of anisotropy in the long range part, with $\lambda_{0}=0$, $\lambda_{\perp}(\mathbf{q})=\lambda_{\perp \rho} q^{-\rho}$ and $\lambda_{\|}(\mathbf{q})=\lambda_{\| \rho} q^{-\rho}$. An anisotropic scaling of the surface $x_{\perp} \rightarrow e^{l} x_{\perp}$ and $x_{\|} \rightarrow e^{l \zeta} x_{\|}$lead to $r_{\lambda} \rightarrow e^{2(1-\zeta) l} r_{\lambda}$. For nonzero $r_{\lambda}$ the scale invariance consequently restricts $\zeta=1$. For $r_{\lambda}=0$, this constraint cannot be imposed and the analytical tractability is lost. To avoid this complexity here, we take $r_{\lambda} \neq 0$ and $\zeta=1$.

The RG procedure follows as before, only a new flow equation for $r_{\lambda}$ is required. The recursion relations are

$$
\frac{d \kappa_{\perp}}{d l}=(z-2) \kappa_{\perp}+\frac{g_{\perp} \kappa_{\perp}}{16 r_{\kappa}^{1 / 2} 2^{\rho}}\left(1-\frac{r_{\lambda}}{r_{\kappa}}\right)+\frac{3 \rho g_{\perp} \kappa_{\perp}}{4 r_{\kappa}^{1 / 2} 2^{\rho}} \frac{\left(r_{\kappa}+r_{\lambda}+2 \sqrt{r_{\kappa}}\right)}{\left(1+\sqrt{r}_{\kappa}\right)^{2}}
$$




$$
\begin{gathered}
\frac{d r_{\kappa}}{d l}=-\frac{g_{\perp} r_{\kappa}^{1 / 2}}{16 \times 2^{\rho}}\left(1-\frac{r_{\lambda}^{2}}{r_{\kappa}^{2}}\right)-\frac{3 \rho g_{\perp} 2^{-\rho}}{4 r_{\kappa}^{1 / 2}(1+\sqrt{r})^{2}}\left(2 r_{\kappa}^{3 / 2}+r_{\kappa}^{2}+r_{\lambda} r_{\kappa}-r_{\lambda}-\frac{r_{\lambda}^{2}}{r_{\kappa}}-\frac{2 r_{\lambda}^{2}}{\sqrt{r}}\right) \\
\frac{d g_{\perp}}{d l}=2 \rho g_{\perp}+\frac{g_{\perp}^{2} 2^{-\rho}}{16 r_{\kappa}^{1 / 2}}\left[\frac{\left(3 r_{\lambda}^{2}+3 r_{\kappa}^{2}+2 r_{\lambda} r_{\kappa}\right)}{r_{\kappa}^{2} 2^{1+\rho}}-3\left(1-\frac{r_{\lambda}}{r_{\kappa}}\right)-36 \rho \frac{r_{\kappa}+r_{\lambda}+2 \sqrt{r_{\kappa}}}{\left(1+\sqrt{r}_{\kappa}\right)^{2}}\right],
\end{gathered}
$$

where $g_{\perp}=\lambda_{\perp}^{2} \Delta K_{2} \kappa_{\perp}^{-3}$. For $r_{\lambda}>0$, Eq. 9 has a fixed point with $r_{\kappa}^{*}>0$, which is a continuation of the isotropic fixed point $r_{\lambda}=r_{\kappa}=1$ for $\rho=0$. The important fixed point for us is $r_{\kappa}^{*}=-r_{\lambda}$, which is physical, from the stability requirement of the surface, only if $r_{\lambda}$ is negative. We consider only this anisotropic case here. The flow equation for $g_{\perp}$ now allows a fixed point, unlike the isotropic case discussed earlier. For small $\rho$, the anisotropic fixed point is at $g_{\perp}^{*} \approx 8 \rho \sqrt{\left|r_{\lambda}\right|}$, with $z=2-\rho / 2+O\left(\rho^{2}\right)$ from Eq. 8 .

The effect of different signs of $\lambda_{\rho}$ is to have opposing (push/pull) effects in the two orthogonal directions. In the KPZ case, they cancel each other producing a EW surface 25]. In the LR case, we predict a new type of rough surface with $z<2$ for $\rho>0$ though on the whole it may be flat but singular with $\chi<0$. Surprisingly, this case seems to be better controlled in the RG approach than the isotropic case. This, in turn, calls for further studies of the $r_{\lambda}=0$ situation entailing anisotropic scaling of space $(\zeta \neq 1)$.

In summary, we have proposed a simple phenomenological model, Eq. 11 that incorporates, as a minimal model, long range interactions in growth problems. We have shown that any interaction decaying slower than $1 / r^{d}$ makes the KPZ or the short range nonlinear case unstable, and asymptotically the surface will have different roughness with exponents depending on the power law of the interaction. The critical behavior in going from a growing to a receding surface can be of various types depending on the dimensionality and the strength of the interaction, as shown in Fig 1 . Power laws decaying faster than $1 / r^{d}$ are suppressed by any local or short range nonlinearity yielding a KPZ like roughness, but when alone, it can produce a still rougher surface.

SM acknowledges support from SFB341. SMB thanks T. Nattermann for hospitality at Köln.

* email: sutapa@thp.uni-koeln.de; sb@iop.ren.nic.in

[1] S. F. Edwards and D. R. Wilkinson, Proc. Roy. Soc. A 381, 17 (1982).

[2] M. Kardar, G. Parisi and Y. C. Zhang, Phys. Rev. Lett. 57, 1810 (1986).

[3] T. Halpin-Healy and Y. -C. Zhang, Phys. Rep. 254, 215 (1995). J. Krug, Adv. Phys. 46, 139 (1997).

[4] A. L. Barbási and H. E. Stanley, Fractal concepts in surface growth (Cambridge University Press, NY, 1995).
[5] Xin-Ya Lei et al, Phys. Rev. E 54, 5298 (1996).

[6] J. Feder and I. Giaever, J. Colloid Interface Sci. 78, 144 (1980).

[7] P. Wojtaszczyk et al, J. Chem. Phys., 99, 7198 (1993); J. Chem. Phys. 103, 8285 (1995).

[8] J. J. Ramsden, Phys. Rev. Letts. 71, 295 (1993).

[9] I. Pagonabarraga and J. M. Rubi, Phys. Rev. Letts. 73, 114 (1994).

[10] I. Pagonabarraga et al, J. Chem. Phys., 105, 7815 (1996).

[11] The nonlinear term chosen does not have a unique sign. It can easily be cured, e.g., by taking the absolute value, but at a cost of complexity. The differnece between the chosen form and a direct coupling of $\mathbf{r}$ and $\mathbf{r}^{\prime}$ is irrelevant for universal behavior. Also not considered in the paper are quenched noise and the driven case.

[12] J. Krug and P. Meakin, Phys. Rev. Letts. 66, 703 (1991) consider a case where nonlocality is introduced in the linear model. This however does not go continuously to the KPZ case - a feature manifest in Eq. 1. Also to be noted is a different proposal, K. B. Lauritsen, Phys. Rev. E 52, R1261 (1995).

[13] M. Kardar, Nucl. Phys. B 290, 582 (1987)

[14] D. A. Huse etal, Phys. Rev. Lett. 54, 2708 (1985)

[15] There is a controversy over the two loop RG result and the fixed point in $d=2$. T. Sun and M. Plischke, Phys. Rev. E 49, 5046 (1994); T. Sun, ibid 51, 6316 (1995); E. Frey and U. C. Tauber, Phys. Rev. E 50, 1024 (1994); 51, 6316 (1995); E. V. Theodorovich, JETP 82, 268 (1996); K. J. Wiese, cond-mat/9706009 reanalyzed all of these two loop calculations.

[16] B. M. Forrest and L. -H. Tang, Phys. Rev. Lett. 64, 1405 (1990)

[17] L. H. Tang etal, Phys. Rev. Lett. 65, 2422 (1990).

[18] C. Doty and J. M. Kosterlitz, Phys. Rev. Lett. 69, 1979 (1992)

[19] S. Mukherji, Phys. Rev E 50 R2407 (1994).

[20] S. Mukherji and S. M. Bhattacharjee, Phys. Rev. B 53, R6002 (1996).

[21] E. Medina et al., Phys. Rev. A 39, 3053 (1989)

[22] That there is no renormalization of $\lambda$ 's can be seen easily in a Martin-Siggia-Rose type field theoretic formulation. (See, e.g., Ref [15], S. Mukherji Phys. Rev. E55, 6459 (1997)). Since the $\lambda$ vertex involves only equal time fields, and for causality the response propagator is nonlocal in time, the triangular diagrams $\left(O\left(\lambda^{3}\right)\right)$ that could have renormalized $\lambda$, vanish identically.

[23] It is important to recognize that the results are even qualitatively different from the case of long range correlated noise in the KPZ equation [21], mainly because the long range part of Eq. 1 does not generate any short range piece under renormalization.

[24] J. Zhang etal, Physica A 189383 (1992)

[25] D. E. Wolf, Phys. Rev. Lett. 671783 (1991). 